\title{
Tercer Puesto ¿QUÉ HACEMOS CON NUESTRO HOTEL?
}

\author{
Fernando Llosa de Cárdenas
}

$\mathrm{L}$

a familia Gil era una de las familias más respetadas del Cusco. El abuelo, Anibal Gil, había nacidoen el seno de un hogar humilde, pero con tesón y trabajo pudo surgir en el campo de la agricultura. Se inició con una pequeña parcela que con el transcurso del tiempo fue creciendo hasta constituir una gran propiedad.

Eustaquio Gil, el hijo, aprendió de su padre el valor de la constancia y la dedicación al trabajo, por lo que desde muy joven trabajo en la hacienda Locamayo, propiedad de la familia ubicada en el valle del Urubamba. A la edad de 35 años, cuando falleció su padre, Eustaquio decidió trasladarse a la ciudad del Cusco y vendió la hacienda a cambio de una cuantiosa suma de dinero.

\section{El hotel}

A fines de la década del 40, el turismo en el Cusco cobro gran impulso debido a la difu- sión mundial del patrimonio arqueológico de csta ciudad y a los trabajos de restauraciónque se estaban emprendiendo. En 1950. Eustaquio decidió construir un hotel de 100 habitaciones en el centro de la ciudad, a dos cuadras de la plaza de armas. Lo llamó Hotel Imperial y lo dotó de todas las comodidades necesarias para que fuera considerado el mejor y el más lujoso del Cusco.

Eustaquio se casó con Inés Yábar, con quien tuvo tres hijos: Esteban, Soraya y Juan Carlos. Mientras Inés dedicaba la mayor parte de su tiempo al cuidado y a la educación de sus hijos, Eustaquio se dedicó por completo al Hotel Imperial. Su carácter, muy vehemente, lo motivó a hacerse cargo personalmente de la administración. incluso fijo su residencia en una suite del hotel.

Con el correr del tiempo, el negocio fue un éxito. Durante los meses de temporada alta, julio y agosto, el hotel alcanzaba casi $100 \%$ de ocupación; llegaba al $70 \%$ en los 
meses intermedios, de abril a junio y de setiembre a diciembre; y se mantenía en $50 \%$ de ocupación durante la temporada baja, de enero a marzo.

El hotel siemprese mantuvo impecable y era común que las reservaciones se efectuaran con mucha anticipación. Se había hecho conexiones con agencias de viajes, líneas aéreas y operadores turísticos para mantener un alto índice de ocupación en las habitaciones, a cambio de un cierto sacrificio en las tarifas. El operador hotelero cede un porcentaje de las ganancias al agente a través del cual se realiza una reservación.

\section{Tiempos difíciles}

En la década del 70, el hotel empezó a experimentar problemas porque sus niveles de ocupación empezaron a descender y. en consecuencia, ya no hubo recursos suficientes para mantenerlo con la calidad que le era tradicional. Por aquellos años, Eustaquio debió enfrentar también la muerte de Inés, su esposa, y los reproches de sus hijos, quienes lo culparon de no haberles dado la debida atención, ni a ella ni al resto de la familia.

Por entonces, Esteban, el hijo mayor, había ingresado al ejército, arma en la que realiz6 una meteórica carrera; Soraya eligí la vida religiosa y al cabo de un tiempo se convirtió en monja misionera; y el hijo menor, Juan Carlos, fue matriculado en el colegio militar a los 15 años.

Eustaquio terminó viviendo solo en el hotel, buscando solucionar los problemas que iban aumentando conforme pasaban los años. Era una persona poco afecta a delegar funciones y se ocupaba directa- mente del manejo administrativo, las fi. nanzas y la contabilidad del hotel.

Mientras tanto, Juan Carlos, después de terminar sus estudios secundarios, es. tudió ingeniería y se volvió empresario. Abrí un taller de carpintería metálica y de madera en Lima, negocio que fue crecien. do poco a poco.

A fines de la década de los ochenta, cl Hotel Imperial pasaba por su peor momento: el país vivía una inflación galopante, había recesión e inestabilidad política y social, además de terrorismo. Todas estas circunstancias provocaron que el turismo descendiera y que el número de turistas extranjeros y de visitantes locales se redujera sustancialmente.

En 1989 el nivel de ocupación promedio del hotel bordeaba el $30 \%$ anual y las deudas empezaban a acumularse. La situación era casi desesperada y Eustaquio estaba ya cansado de cargar con el peso de la responsabilidad. Sin embargo, su situación familiar estaba profundamente deteriorada; a sus hijos varones no los veía desde hacía dos años, y de Soraya, que estaba en una misión en Centroamérica, no tenía noticias desde hacía cinco años.

Los hijos varones vivían distanciados entre sí, ya que Esteban estuvo involucrado con el gobierno militar, primero, $y$ con el gobierno aprista, después, con los cuales Juan Carlos tuvo siempre profundas discrepancias.

En 1990, con la asunción al poder del ingeniero Fujimori, se empezaron a advertir algunas señales de cambio; sin embargo, los primeros signos de recuperación recién se mostraron en 1993. El hotel empezó a levantar su nivel de ingresos. lo aue 
le permitio mantenerse sin efectuar mayores inversiones.

A fines de 1995, Eustaquio Gil falleció dejando un hotel antiguo con la mayor parte de sus instalaciones deterioradas y sus cuentas en desorden. Los estados financieros habían sido hechos s6lo con propósitos de tributación (ver Anexo l), pero no reflejaban ta realidad de la empresa. Tampoco se sabía mucho sobre las fuentes de ingreso y los costos de operación del hotel.

\section{El futuro}

A raíz del fallecimiento del propietario y del auge que estaba recobrando el turismo en la ciudad del Cusco, los herederos recibieron varias propuestas de compra, alquiler e incluso explotación en asociación. El hotel era muy atractivo por sus dimensiones, su cercanía a la plaza de armas y porque no había vestigios arqueológicos en su interior, razón por la que podía ser remodelado en armonía con su entorno, de acuerdo con las disposiciones locales.

Esteban y Juan Carlos decidieron reunirse para decidir el futuro del hotel y analizar las distintas ofertas que les habian presentado. Soraya les comunicó que estaría de acuerdo con lo que ellos dispusieran.

Las opciones que tenían los hermanos eran las siguientes:

- Vender. El valor comercial del hotel era de 5 millones de dólares.

- Alquilar. La empresa Quality International había manifestado su interés en alquilar el hotel durante 20 años por un valor anual de 400 mil dólares, cifra que se iría actualizando anualmente de acuerdo con la inflación del dólar. Después de este plazo, la propiedad retornaría a sus dueños con todas las mejoras que se hubieran realizado.

- Operar el hotel. Juan Carlos pensaba que existía una buena oportunidad de operar el negocio, ya fuera solos o en asociación, pues el turismo mostraba un crecimiento continuo en los últimos años y era intención del Gobierno seguir estimulando este sector (ver Anexo 2).

-Asociación en participación. La empresa Interamerican Investment había sugerido una asociación en participación por la cual los hermanos Gil aportarian el hotel; y la empresa, la inversión necesaria para la modernizacion y posterior administración de éste. A cambio, la empresa entregaría a los propietarios el $7 \%$ de las utilidades netas (después de cubiertos costos operativos, administrativos, financieros y fiscales). Interamerican Investment manifestaba haber obtenido condiciones muy convenientes para financiar el $50 \%$ de la inversión: 10 años de plazo, 2 años de gracia y una tasa de $8,5 \%$ de interés anual en dólares.

Los hermanos Gil se reunieron el 3 de febrero de 1996. Mientras Esteban quería vender el hotel a su valor comercial para, luego de pagar las deudas, distribuir el dinero entre los tres hermanos, Juan Carlos quería potenciar el negocio invirtiendo en su modernización. Pensaba que así se podría alcanzar los estándares de un hotel de categorfa y lograr un mayor ingreso que el que obtendrían con la liquidación, el alquiler o la asociación en participación.

Esteban, que se había documentado en la materia, pensaba que el sector hotelero 
era muy riesgoso (ver Anexo 3), y no estaba dispuesto a arriesgar su dinero en un campo que no conocía. La discusión se prolongó por horas y parecía que no se llegaría a acuerdo alguno, hasta que Juan Carlos, al darse cuenta de que no iba a convencer a su hermano, sugirió contratar a un consultor externo especialista en el campo de la hotelería, con el fin que hiciera un estudio de mercado, un análisis de la inversión necesaria para modernizar el hotel en función de la demanda del mercado y estimara los costos de operación y administración. De esta manera podrían comparar esta opción con las otras tres. Esteban aceptó la idea de su hermano siempre y cuando se contratara a un consultor internacional de reconocido prestigio.

Luego de las indagaciones del caso, los hermanos Gil contrataron a Sally Hospitality, empresa reconocida en el mercado y cuyos honorarios, 35 mil dólares, estaban por debajo de los de otras empresas consultadas.

Tiempo después, el 4 de junio, Sally Hospitality presentó un informe (ver Anexo
4) que Esteban y Juan Carlos estudiaron con detenimiento. Grande fue su desilusión, sin embargo, cuando vieron que el estudio presentaba una serie de números y porcentajes que los llenaba de incertidumbre. Advirtieron entonces que tomar la decisión no les sería tan sencillo. ¿Debían asumir un escenario optimista o pesimista?, ¿las ganancias serían las suficientes como para justificar el riesgo? Estas respuestas no estaban escritas en el estudio.

Los hermanos Gil decidieron entonces recurrir a GLL Consulting para que efectuara el análisis financiero de las alternativas posibles. Precisamente, GLL era una empresa recién formada para efectuar consultoría financiera en proyectos de inversión con especial énfasis en el sector hotelero. Sus fundadores eran todos magíster en Administración de ESAN, habían estudiado profundamente el sector e identificado cinco factores claves para que un proyecto hotelero tuviera éxito (ver Anexo 5).

Esteban y Juan Carlos acordaron esperar los resultados del estudio de estos jóvenes profesionales. 


\section{¿QUÉ HACEMOS CON NUESTRO HOTEL?}

\section{Anexos}

1. Estados financieros del hotel Imperial

2. La industria turística hotelera en el mundo

3. El riesgo en la industria turística

4. Resumen del informe de Sally Hospitality

5. Factores claves para el éxito en un proyecto hotelero 
Anexo 1

ESTADOS FINANCIEROS DEL HOTEL IMPERIAL

\begin{tabular}{|c|c|c|c|}
\hline \multicolumn{4}{|c|}{ BALANCE AL 31 DE DICIEMBRE DE 1994} \\
\hline \multicolumn{2}{|c|}{ Letivo } & & \\
\hline \multicolumn{2}{|l|}{ Activo circulante } & \multicolumn{2}{|l|}{ Pasivo a corto plazo } \\
\hline Efectivo & 150.000 & Cuentas por pagar & 55.000 \\
\hline Cuentas por cobrar & 35.000 & Documentos por pagar & 25.000 \\
\hline Documentos por cobrar & 30.000 & Intereses por pagar & 18.000 \\
\hline Almacén de materiales & 25.000 & Impuestos por pagar & 53.000 \\
\hline Gastos pagados por adelantado & 5.000 & Total pasivos a corto plazo & 151.000 \\
\hline \multicolumn{4}{|c|}{ Total activo circulante $\quad 245.000$} \\
\hline Activo no circulante & & Pasivo a largo plazo & 245000 \\
\hline $\begin{array}{l}\text { Activo no circulante } \\
\text { Activo fijo }\end{array}$ & & $\begin{array}{l}\text { Obligaciones } \\
\text { Hipoteca }\end{array}$ & $\begin{array}{l}245.000 \\
185,000\end{array}$ \\
\hline $\begin{array}{l}\text { Actuvo tijo } \\
\text { Terreno }\end{array}$ & 2.000 .000 & $\begin{array}{l}\text { Hipoteca } \\
\text { Préstamo a largo olazo }\end{array}$ & 47000 \\
\hline \multirow{2}{*}{\multicolumn{4}{|c|}{$\begin{array}{ll}\text { Edificio - depreciación acumu- } & \text { Total pasivos a corto plazo } \\
\text { lada }\end{array}$}} \\
\hline & & & \\
\hline \multicolumn{4}{|c|}{ Equipo-depreciacion acumulada 235.000} \\
\hline \multirow{2}{*}{\multicolumn{4}{|c|}{$\begin{array}{lrll}\text { Total activo fijo } & 2.805 .000 & \text { Total de pasivos } & \mathbf{6 2 8 . 0 0 0} \\
\text { Seguros pagados por adelantado } & 47.000 & \end{array}$}} \\
\hline & & & \\
\hline \multirow[t]{3}{*}{ Total activo no circulante } & 2.852 .000 & Patrimonio & 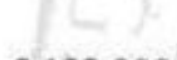 \\
\hline & & Capital social & $2,120.000$ \\
\hline & & Utilidades retenidas & 349.000 \\
\hline \multicolumn{4}{|c|}{ Total de patrimonio } \\
\hline
\end{tabular}

\section{ESTADO DE PÉRDIDAS Y GANANCIAS Al 31 de diciembre de 1994}

Ventas netas

1.025 .000

Costo de ventas

697.000

Utilidad bruta

328.000

Depreciación

95.000

Otros costos fijos

78.000

Ganancias antes de impuestos

155.000

Impuestos

46.500

Utilidad neta

108.500 


\section{Anexo 2 \\ LA INDUSTRIA TURÍSTICA Y HOTELERA EN EL MUNDO}

\begin{abstract}
En el mundo
El turismo es una actividad trascendente para la economía mundial. Capta el $25 \%$ de los ingresos de todo el sector servicios y ha mostrado un incremento considerable en los últimos 30 años. Es también la mayor fuente de recaudación de impuestos en el ámbito mundial y la mayor empleadora después de la agricultura, razón por la cual constituye una fuente de progreso para muchas regiones que poseen belleza natural o riqueza cultural; nuestro país cuenta con muchos recursos de ambos tipos.
\end{abstract}

En los últimos años son muchos los países que están promoviendo esta industria debido a su capacidad de generación de divisas y de empleo $y$ a su efecto multiplicador sobre el resto de la economía, como transporte, agricultura, pesca, alimentos, construcción, servicios de mantenimiento y financieros, entre otros. Los residentes locales se benefician también con la infraestructura básica que se debe construir para atender al turismo, como medios de transporte, redes de agua y desague, electricidad, servicios recreacionales y de salud, etc.

El desarrollo de hoteles de ciudad es muy importante en mercados emergentes y econo. mías en transición, pues estos establecimientos brindan infraestructura básica y servicios de hospedaje con estándares internacionales a los hombres de negocios e inversionistas que visitan la región. Se han convertido así en un factor que contribuye a promover el crecimiento económico. El flujo de hombres de negocios permanece más o menos estable a lo largo del año, por lo que genera flujos de dinero relativamente estables. Esto suele atraer competidores y obliga a mantener las tarifas en un nivel que no puede ser muy elevado.

Los hoteles de tipo vacacional están dirigidos a otro mercado, constituido principalmente por personas que buscan descansar, visitar atractivos turísticos o apreciar la belleza del paisaje. El flujo de este tipo de turismo se concentra en determinadas épocas del año, razón por la cual el negocio tiene un fuerte componente estacional y los flujos de dinero son muy variables. Esto hace que en los meses de alta temporada se tenga que acumular ganancias para cubrir las épocas de baja temporada. En este tipo de hoteles existen menos competidores.

\section{En el Perú}

Durante los primeros 7 meses de 1996 visitaron el pais 364.145 turistas extranjeros. Proyectando esta cifra, se estima que a fin de año se puede obtener por este concepto un ingreso de divisas de aproximadamente 650 millones de dólares, los que tendrán un gran impacto sobre la economia. Las estadísticas señalan que el turista extranjero gasta en promedio 1.050 dólares durante su estada en el pafs. El gran crecimicnto que experimenta la industria en los últimos af̂os ha sido una de las causas del crecimiento observado en el empleo, con una mínima inversión.

\begin{tabular}{|rrr|}
\hline Anio & Turistas & $\begin{array}{r}\text { Crecimiento } \\
\text { Porcential }\end{array}$ \\
\hline 1992 & 216.534 & \\
1993 & 271.901 & 25,57 \\
1994 & 386.120 & 42,01 \\
1995 & 485.169 & 25,65 \\
1996 & $364.145^{*}$ & $28,67 \%$ \\
\hline
\end{tabular}

* A julio de 1996.

Si bien la mayoría de turistas vienen atraidos por Cusco y Machu Picchu, se está desarrollando una serie de destinos turísticos alternativos, como Lima, Arequipa, Iquitos, Huaraz (turismo de aventura) y Madre de Dios (ecoturismo) para lograr que el visitante extienda su tiempo de permanencia en el país y, por lotanto, aumente su gasto. Para alcanzar este objetivo, así como para mantener o incrementar la gran 
tasa de crecimiento del sector, es necesario desarrollar una serie de inversiones en el campo del turismo y la hostelería. El Gobierno está a punto de promulgar la nueva Ley General de Turismo.

\section{Anexo 3 \\ EL RIESGO EN LA INDUSTRIA TURÍSTICA}

A pesar de sus grandes proyecciones de crecimiento y el gran impacto que tiene sobre el resto de la economía, la hostelería es una actividad muy riesgosa, por lo que las inversiones en este campo deben estudiarse profundamente. Los principales riesgos a los que está sujeta la hostelería son los siguientes:

- Tiene un alto componente de costo fijo debido al elevado capital invertido para la adquisición del terreno, la construcción, el equipamiento, etc. También demanda altos costos de operación, por el gran número de personal empleado y el capital de trabajo necesario.

- Demora en la implementación del proyecto, ya sea por inconvenientes en la construcción, la obtención de licencias, el suministro y/o la importación de materiales o debido a problemas en la operación. Todo esto origina dificultades en el pago de la deuda y obliga a reprogramarla.

- Brinda un servicio que no se puede almacenar para ser vendido después. Los ingresos no percibidos por una noche de alojamiento no vendida se pierden para siempre.
- Posibilidad que el producto ofrecido no encaje con el mercado o con los servicios que el operador pueda ofrecer.

-Posibilidad de sobreoferta que genere una guerra de precios y reduzca sustancialmente la utilidad del negocio.

- Posibilidad que aumenten los costos operativos por encima de las tarifas que el mercado pueda absorber.

- Múltiples factores que pueden afectar el nivel de ocupación de los hoteles, como: desórdenes políticos, terrorismo, problemas de seguridad, cambios en la legislación, inestabilidad económica, variaciones en el tipo de cambio, inflación, recesión. También cambios en la moda, los hábitos de consumo o el poder adquisitivo de los visitantes; estacionalidad; cambio en el entorno de los negocios; variación en los costos de los viajes o en el interés de los operadores turísticos en atender determinado mercado; cambio en las rutas de transporte; problemas naturales como terremotos, huracanes o erosión del terreno; problemas ambientales, como polución, ruido, etc. 


\section{Anexo 4 \\ RESUMEN DEL INFORME DE SALLY HOSPITALITY}

\section{Análisis de mercado}

Se efectuó un estudio de los hoteles de mayor categoría de la ciudad, de los que serian comparables al Hotel Imperial.

Primera categorfa:

Hotel San Martín 110 habitaciones

Santa Catalina

89 habitaciones

Segunda categorfa

Internacional

La Quebrada

125 habitaciones

Sacsayhuamán

Cora Cora

Embasy

Total

108 habitaciones

85 habitaciones

73 habitaciones

70 habitaciones

660 habitaciones

El promedio de ocupación que obtuvieron estos hoteles durante 1995 fue de $72 \%$, con una tarifa promedio de 80 dólares por noche.

Debido a la estabilidad económica y política que ha alcanzado el pars, los turistas y hombres de negocios están regresando a visitar la ciudad. El número de visitantes está en aumento, por lo que se percibe que estos niveles de ocupación se elevarán, aunque se estén construyendo dos hoteles más que estarian comprendidos dentro de estas categorias.

\section{Hoteles en construcción}

$\begin{array}{lr}\text { El oro } & 115 \text { habitaciones } \\ \text { San Paolo } & 78 \text { habitaciones } \\ \text { Total } & 193 \text { habitaciones }\end{array}$

La mayoría de los hoteles existentes son antiguos y no ofrecen las comodidades necesarias para un viajero del mercado superior. Mediante encuestas se determinó que el $45 \%$ de los huéspedes de estos hoteles se alojarían en otros de mayor categoría si estuvieran disponibles, a pesar de que el precio fuera mayor. Por esta razón, puede afirmarse que existe mercado para el desarrollo de un hotel de categoría internacional en la ciudad del Cusco.

Si se remodela el Hotel Imperial dándole categoría internacional, se podría alcanzar un promedio de ocupación anual de $75 \%$ a una tarifa de 130 dólares por noche. Para llegar a esta cifra, el hotel tendría que ser gerenciado por una cadena internacional que maneje una red de reservaciones a nivel mundial. Además, tendría que orientarse hacia el mercado objetivo. Según encuestas realizadas, el $90 \%$ de los visitantes que llegan al Cusco lo hace por turismo; sólo un $10 \%$ lo hace por negocios. Por este motivo, seria recomendable enfocar el hotel hacia el mercado turístico, pero sin descuidar la posibilidad de atender al mercado de negocios.

\section{Análisis de ubicación}

La ubicación del hotel es ideal, pues está en una zona céntrica, atractiva y de fácil acceso. El layout del hotel es susceptible de ser modificado. Para distribuir los costos fijos entre un mayor numero de habitaciones, es factible ampliar el número de éstas a 140 mediante al gunas modificaciones en los cuatro pisos del hotel.

\section{Análisis de ocupación}

Seprevé que la construcción del hotel demorará un año, periodo durante el cual no se percibirá ingreso alguno. Durante el primer año de operación se tiene previsto alcanzar un nivel de ocupación de $60 \%$, que irá aumentando a $68 \%$ y $72 \%$ en los años sucesivos y finalmente se estabilizará en $75 \%$.

\section{Inversión necesaria}

Para efectuar las modificaciones necesarias con el objeto de darle categoría internacional al 
hotel, se requiere realizar las siguientes inversiones:

$$
\begin{array}{lr}
\text { Ingenieria } & \text { USS } \\
250.000 \\
\text { Obra } & 3.546 .000 \\
\text { Equipamiento de edificio } & 1.087 .000 \\
\text { Equipamiento hotelero } & 1.811 .000 \\
\text { Total parcial } & \text { US } \$ 6.694 .000
\end{array}
$$

\section{Ingresos}

Sobre la base del estudio de varios hoteles de categoría semejante al que tendría el Hotel Imperial, se ha estimado la distribución del total de ingresos.

$\begin{array}{llrl}\text { Hospedaje } & \text { entre } 73 \text { y } 77 \% \\ \text { Alimentos y bebidas entre } 18 \text { y } 20 \% \\ \text { Teléfonos } & \text { entre } 1,5 \text { y } 2,5 \% \\ \text { Otros operativos } & \text { entre } 2 \text { y } 2,5 \% \\ & & & \\ & \end{array}$

En promedio, se espera que el total de egresos antes del pago de amortizaciones, intereses e impuestos esté comprendido entre el 60 y el $65 \%$ del total de ingresos del hotel. Los egresos de un proyecto hotelero se pueden dividir en cuatro tipos según su fuente:

a) Egresos operativos. Se han considerado como un porcentaje de cada uno de los ingresos obtenidos por la operación de un hotel. Se estima que en conjunto representen del 20 al $27 \%$ del total de ingresos e individualmente estén dentro de los siguientes porcentajes del total de ingresos:

$\begin{array}{ll}\text { Hospedaje } & \text { entre } 15 \text { y } 18 \% \\ \text { Alimentos y bebidas entre } 40 \text { y } 50 \% \\ \text { Telefonos } & \text { entre } 45 \text { y } 50 \% \\ \text { Otros operativos } & \text { entre } 60 \text { y } 70 \%\end{array}$

b) Egresos generales. Se han considerado como un porcentaje de los ingresos totales. Se estima que en conjunto representen entre el 20 y el $25 \%$ del total de ingresos e individualmente estén dentro de los siguientes

porcentajes del total de ingresos:

$\begin{array}{llr}\text { Administración } & \text { entre } & 6 \text { y } 10 \% \\ \text { Publicidad (marketing) } & \text { entre } & 3 \text { y } 7 \% \\ \text { Energía } & \text { entre } & 3,5 \text { y } 5 \% \\ \text { Mantenimiento } & \text { entre } & 3,5 \text { y } 4,5 \%\end{array}$

c) Otros egresos. En esta partida están considerados los impuestos de operación, licencias de funcionamiento, pago de arbitrios y otros, así como los seguros del inmucble durante su opcración. Se han estimado como porcentaje delos ingresostotales. Se estimaque en conjun. to representen entre el 1 y el $5 \%$ del total de ingresos e individualmente estén dentro de los siguientes porcentajes del total de ingresos:

Impuestos

Seguros

entre 0,5 y $5 \%$ entre 0.4 y $1 \%$

d) Participaciones. Están considerados los costos preoperativos y los costos de usar una cadena internacional que administre el hotel. Dentro de los costos preoperativos están considerados el entrenamiento del personal y el capital de trabajo, estimadosen 200 mil dolares. Los costos de administración pueden ser estimados como un porcentaje de los ingresos totales. Se estima que en conjunto representen entre el 6 y el $10 \%$ del total de ingresos $\mathrm{e}$ individualmente estén dentro de los siguientes porcentajes del total de ingresos:

$\begin{array}{ll}\text { Management fee: } & \text { entre } 3 \text { y } 5 \% \\ \text { Incentive fee: } & \text { entre } 1 \text { y } 3.5 \% \\ \text { License fee: } & \text { entre } 0 \text { y } 4 \%\end{array}$

Es muy importante identificar un operador adecuado para el éxito del proyecto hotelero. Es factible que se pacte con éste el pago de solo dos tarifas de operación:

\section{Management fee: \\ $4 \%$ del total de Ingresos \\ Incentive fee: \\ $10 \%$ del GOP (ingresos operativos netos)}

Si se negocia adecuadamente con el operador, el license fee puede no ser considerado. Asimismo, debe buscarse que el pago del in. 
centive fee se difiera o subordine al pago de la deuda, es decir, que se tome como base los costos netos de operación después del servicio de la deuda.

Es deseable alentar la inversión del operador con una participación de por lo menos $10 \%$, como una señal de buena voluntad al entablar una relación de largo plazo y con el fin que el operador se identifique más con el éxito de la empresa.

\section{Financiación}

Dada la naturaleza de la actividad, para hacer viable el proyecto se requiere de un periodo alto de amortización con periodos adecuados de gracia. La comunidad financiera internacional ha sido generalmente renuente a proveer este tipo de financiación.

Para apalancar adecuadamente un proyecto se requiere un plazo de por lo menos 7 afos de pago con 2 años de gracia.

Es posible conseguir capital a una tasa de entre 8 y $10 \%$ por intermedio de una fuente internacional de financiamiento y con una re- lación Deuda/Capital igual a 1 ó 1,5 como máximo.

\section{Regulaciones legales}

El D. L. 662 establece el marco legal básico para las inversiones extranjeras en el Perú. En esta disposición se garantiza al inversionista extranjero estabilidad tributaria y juridica y libre movilidad de capitalcs, esto es, puede expatriar libremente su capital o utilidades. Además se establece un trato igualitario entre inversionistas nacionales y extranjeros.

Las utilidades están gravadas con el $30 \%$ de impuesto a la renta, pero el Gobierno ha otorgado incentivos para promover las inversiones en el sector turismo. Los nuevos hoteles están exentos del pago del impuesto mínimo a la renta $\mathrm{y}$ del impuesto al patrimonio predial.

Adicionalmente, para empresas que inicien su operación antes del 31 de diciembre de 1998. la tasa de depreciación anual vigente de $3 \%$ se ha modificado a $10 \%$, durante los tres primeros años de operación si las propiedades se encuentran en Lima, o durante los cinco primeros años si están ubicadas en provincias. 


\section{FACTORES CLAVES PARA EL ÉXITO EN UN PROYECTO HOTELERO}

\section{Buena ubicación}

La ubicación es un aspecto fundamental para el éxito de un proyecto. Es necesario identificar una zona que sea, o en el futuro pueda ser. un mercado conveniente para el desarrollo de un proyecto hotelero, y dentro de esta zona adquirir un terreno aparente que sea de fácil acceso, esté próximo a lugares de interés para el cliente, tenga una vecindad agradable y otras características que demande el público al que se pretende servir.

\section{Operador internacional}

Asociarse con un operador internacional de solida reputación incrementa la probabilidad de éxito de un proyecto por varios motivos:

a) Por lo general, estas empresas conocen muy bien el negocio y estudian el proyecto en profundidad antes de tomar parte en él, lo que reduce el alto riesgo inherente a esta actividad.

b) Los operadores internacionales cuentan con una red internacional de marketing que ayudará a elevar el nivel de ocupación del establecimiento.

c) Los operadores internacionales conocen muy bien la forma de operar el negocio, saben lo que el público quiere y cómo lograrlo con el mínimo costo.

d) Se generan cconomías de escala y si- e) Quienes viajan por negocios se inclinan más por hoteles conocidos internacionalmente que ofrecen servicios globales.

\section{Buen servicio}

Si el cliente no queda satisfecho con el servicio es muy probable que no regrese al hotel y, además, que realice publicidad adversa y presente reclamos ante la agencia o el operador a través del cual efectuó su reserva.

\section{Buen producto}

Debe desarrollarse un producto adecuado para el cliente objetivo. Por esta razón es muy necesario realizar un estudio de mercado con la finalidad de identificar al cliente, sus necesidades y lo que está dispuesto a pagar por satisfacerlas.

\section{Precio adecuado}

Se tiene que analizar si el negocio es viable con lo que el cliente está dispuesto a pagar. Si el mercado no puede absorber un precio elevado, se tendrá que reducir las tarifas. $\mathrm{Si}$ el mercado puede absorber un nivel de precios alto, la competencia no tardará en penetrar en el mercado y posiblemente se generará una guerra de precios que no beneficiará a competidor alguno. 